\title{
UNCONDITIONAL CONVERGENCE OF SOME CRANK-NICOLSON LOD METHODS FOR INITIAL-BOUNDARY VALUE PROBLEMS
}

\author{
WILLEM HUNDSDORFER
}

\begin{abstract}
In this paper convergence properties are discussed for some locally one-dimensional (LOD) splitting methods applied to linear parabolic initialboundary value problems. We shall consider unconditional convergence, where both the stepsize in time and the meshwidth in space tend to zero, independently of each other.
\end{abstract}

\section{INTRODUCTION}

In this paper the accuracy of some simple splitting methods will be analyzed. The methods are used for the numerical solution of initial-boundary value problems for partial differential equations (PDE's) in two space dimensions. Discretization in space of such PDE problems leads to a large system of ordinary differential equations (ODE's)

$$
\dot{u}(t)=F(t, u(t)) \quad(0 \leq t \leq T), \quad u(0)=u_{0},
$$

where the vector function $F$ contains discretized space derivatives. It is often possible to decompose $F$ into two simpler functions $F_{1}$ and $F_{2}$,

$$
F(t, v)=F_{1}(t, v)+F_{2}(t, v) \text {. }
$$

Standard implicit methods to approximate (1.1) require the solution of large systems of algebraic equations involving the whole function $F$. A well-known method is the implicit midpoint rule

$$
u_{n+1}=u_{n}+\tau F\left(t_{n}+\frac{1}{2} \tau, \frac{1}{2} u_{n}+\frac{1}{2} u_{n+1}\right) \quad(n=0,1,2, \ldots) .
$$

For linear problems, (1.3) is also often called the Crank-Nicolson method in the PDE literature. The vectors $u_{n}$ approximate the exact solution $u$ of (1.1) at $t_{n}=n \tau$ with $\tau>0$ the stepsize in time. Method (1.3) is of 2 nd order in the classical ODE sense.

In terms of computational effort it can be more attractive to exploit the splitting (1.2). In this paper we shall consider some locally one-dimensional (LOD) methods, where the step $u_{n} \mapsto u_{n+1}$ is performed in two stages, in each of which only one of the functions $F_{1}$ or $F_{2}$ is used. The best known LOD method is

Received July 12, 1990.

1991 Mathematics Subject Classification. Primary 65M12, 65M15, 65M20.

Key words and phrases. Numerical analysis, initial-boundary value problems, LOD methods, unconditional convergence. 
based on the backward Euler method (see Yanenko [17]). To achieve 2nd order, Yanenko also derived LOD methods based on the Crank-Nicolson method. In this paper we primarily consider

$$
\begin{gathered}
u_{n+1 / 2}=u_{n}+\tau F_{1}\left(t_{n}+\frac{1}{4} \tau, \frac{1}{2} u_{n}+\frac{1}{2} u_{n+1 / 2}\right), \\
u_{n+1}=u_{n+1 / 2}+\tau F_{2}\left(t_{n}+\frac{3}{4} \tau, \frac{1}{2} u_{n+1 / 2}+\frac{1}{2} u_{n+1}\right)
\end{gathered}
$$

for $n \geq 0$. The vector $u_{n+1 / 2}$ is an intermediate vector, only used for the internal computation. If $F_{1}$ and $F_{2}$ have a more simple structure than $F$, the computation of $u_{n+1}$ from (1.4) can be done more efficiently than from (1.3). However, the LOD method (1.4) will have 2 nd order only if $F_{1}, F_{2}$ are linear and commuting; in more general situations it will be merely of 1 st order, owing to lack of symmetry (see [4]). Below we will consider two modifications which restore symmetry and 2 nd order.

Denote (1.4) as

$$
u_{n+1}=\Phi_{1,2}\left(u_{n}\right) \quad(n=0,1,2, \ldots),
$$

where the order of the indices 1,2 refers to the fact that first $F_{1}$ was used and subsequently $F_{2}$. In this paper we shall call (1.5) the basic scheme.

Symmetry can be restored by interchanging after each step $F_{1}$ and $F_{2}$. This idea, which can be found in $[9,10]$, leads to the following modification:

$$
u_{n+1}=\Phi_{1,2}\left(u_{n}\right), \quad u_{n+2}=\Phi_{2,1}\left(u_{n+1}\right) \quad(n=0,2,4, \ldots),
$$

which will be called here the sequentially alternating scheme. This modification indeed has again 2 nd order, and it requires the same amount of computational work as (1.5).

At first sight the scheme (1.6) seems superior to (1.5). This conclusion, however, will turn out not to be justified. The reason is that the classical order concept for ODE's to which we referred to until now is the order of consistency/convergence for nonstiff ODE's, where $F$ satisfies a Lipschitz condition with a moderate Lipschitz constant $L$, and $\tau L$ is assumed to be sufficiently small. In our situation, where (1.1) originates from a PDE problem, the Lipschitz constant $L$ will contain negative powers of the meshwidth in space $h$. As a consequence, $L$ will be very large for fine space grids, and the classical convergence theory cannot be applied. In fact, the order of the discretization errors in time may be affected by small meshwidths, a phenomenon called order reduction. We will see that order reduction destroys the favorable convergence properties that (1.6) has for nonstiff ODE's.

Another modification of (1.5), due to Swayne [14], reads

$$
u_{n+1}=\frac{1}{2} \Phi_{1,2}\left(u_{n}\right)+\frac{1}{2} \Phi_{2,1}\left(u_{n}\right) \quad(n=0,1,2, \ldots) .
$$

We shall refer to (1.7) as the parallel alternating scheme. Its order of consistency/convergence in the classical ODE sense is also 2. The results in [14] might give the impression that this scheme does not suffer from order reduction. It will be shown that this is not entirely correct: the order of the local discretization errors is reduced for small $h>0$, but it will also be shown that in the transition from local to global errors this reduction is annihilated because of damping and cancellation effects. 
The accuracy analysis for the above LOD methods will be performed on simple linear heat flow problems with a source term and Dirichlet boundary conditions. For space discretization, standard finite differences on a rectangular grid $\Omega_{h}$ are considered, $h$ being the meshwidth in space. To obtain a full convergence analysis, we let $\tau$ and $h$ tend to zero simultaneously. The effect of $h \downarrow 0$ on the orders in time is summarized by Tables 1.1 and 1.2. Only for fixed $h=h_{0}>0$ does the classical ODE theory apply.

TABLE 1.1

Orders of consistency in time

\begin{tabular}{|c|c|c|c|}
\hline method & $(1.5)$ & $(1.6)$ & $(1.7)$ \\
\hline$h=h_{0}$ & 1 & 2 & 2 \\
$h \downarrow 0$ & $\frac{1}{4}$ & $\frac{1}{4}$ & $\frac{5}{4}$ \\
\hline
\end{tabular}

TABLE 1.2

Orders of convergence in time

\begin{tabular}{|c|c|c|c|}
\hline method & $(1.5)$ & $(1.6)$ & $(1.7)$ \\
\hline$h=h_{0}$ & 1 & 2 & 2 \\
$h \downarrow 0$ & 1 & {$\left[\frac{1}{4}, \frac{1}{2}\right]$} & 2 \\
\hline
\end{tabular}

The entry $\left[\frac{1}{4}, \frac{1}{2}\right]$ for method (1.6) in the second table means that the order of convergence is in the interval $\left[\frac{1}{4}, \frac{1}{2}\right]$. These results are valid for $L_{2}$-norms. (The convergence of method (1.6) in the maximum norm is even worse; see Table 5.3.)

The fact that LOD schemes may suffer from order reduction as $h \downarrow 0$ was mentioned already in [17] for a heat equation without source term but with boundary conditions varying in time. For such problems boundary correction techniques to restore the order of consistency are known for the basic scheme (1.5) (see [8]). In some counterexamples we will see that the order reduction is also present for homogeneous boundary conditions if there is a source term in the differential equation. Since many practical problems can be modelled with homogeneous boundary conditions, but with a function $F$ which is nonlinear, or at least affine, this point should be taken into account in the derivation of boundary correction techniques. Observe from Table 1.2 that as far as the order of convergence is concerned, such corrections are not necessary for (1.5), (1.7); for these two schemes the orders of convergence in time are not reduced as $h \downarrow 0$. Still, boundary corrections may be useful to obtain smaller error constants (see for instance the numerical results in [13] for the LOD method based on the backward Euler method, which is convergent with order 1 for both cases $h$ fixed and $h \downarrow 0$ [11]).

Although we shall deal in this paper only with simple linear problems with two space dimensions, it should be noted that the LOD schemes are stable for much more general problems, nonlinear and with arbitrarily many space dimensions (see [15]).

The linear model problem used for the analysis of the LOD methods is described in $\S 2$. The accuracy analysis of the methods can be found in $\S \S 3,4$, and 5. This analysis is closely related to the one given in [6] for the PeacemanRachford ADI method. Method (1.5) is included in these sections, in spite of the fact that this method is already known not to have 2nd order, not even for fixed ODE's. It will turn out that the convergence proof for (1.5) contains some basic ideas and derivations which are also applicable to the other methods.

It is also possible to construct a scheme of the type (1.4), starting from the 
trapezoidal rule instead of the implicit midpoint rule, or to use in (1.4) not the time levels $t_{n}+\frac{1}{4} \tau, t_{n}+\frac{3}{4} \tau$ but only $t_{n}+\frac{1}{2} \tau$, for example. In $\S 6$ it will be briefly discussed to what extent such modifications would affect the convergence results. $\S 7$ contains general concluding remarks.

\section{Preliminaries}

2.1. The model problem. We shall consider application of the LOD methods to parabolic model problems on the unit rectangle $\Omega=(0,1)^{2}$ with $0 \leq t \leq T$,

$$
\begin{aligned}
& \frac{\partial}{\partial t} \mathbf{u}(x, y, t)=\Delta \mathbf{u}(x, y, t)+\mathbf{g}(x, y, t) \quad \text { on } \Omega, \\
& \mathbf{u}(x, y, t)=\mathbf{u}_{\Gamma}(x, y, t) \quad \text { on } \Gamma=\partial \Omega, \\
& \mathbf{u}(x, y, 0)=\mathbf{u}_{0}(x, y) \text { on } \Omega \cup \Gamma .
\end{aligned}
$$

Let $\Omega_{h}$ be the spatial grid $\left\{\left(x_{i}, y_{j}\right): x_{i}=i h, y_{j}=j h, 1 \leq i, j \leq m\right\}$ with $h=1 /(m+1)$. Grid functions on $\Omega_{h}$ will be identified in the usual way with vectors in $\mathbb{R}^{M}, M=m^{2}$, assuming row-wise ordering on $\Omega_{h}$. Thus, $w: \Omega_{h} \rightarrow \mathbb{R}$ will also be written as

$$
w=\left[\begin{array}{c}
w_{1} \\
w_{2} \\
\vdots \\
w_{m}
\end{array}\right] \in \mathbb{R}^{M} \quad \text { with } w_{j}=\left[\begin{array}{c}
w_{1 j} \\
w_{2 j} \\
\vdots \\
w_{m j}
\end{array}\right] \in \mathbb{R}^{m}, w_{i j}=w\left(x_{i}, y_{j}\right)
$$

Standard 2nd-order finite difference discretization of the Laplacian $\Delta$ on $\Omega_{h}$ leads to the semidiscrete system

$$
\dot{u}(t)=A u(t)+f(t) \quad(0 \leq t \leq T), \quad u(0)=u_{0} .
$$

The vector $u(t) \in \mathbb{R}^{M}$ has components $u_{i j}(t) \approx \mathbf{u}\left(x_{i}, y_{j}, t\right)$, and $A=A_{1}+A_{2}$ with $A_{1}, A_{2} \in L\left(\mathbb{R}^{M}\right)$ approximating $\partial^{2} / \partial x^{2}$ and $\partial^{2} / \partial y^{2}$, respectively, as given by the stencils

$$
A \cong h^{-2}\left[\begin{array}{rrr}
1 & 1 & \\
1 & -4 & 1 \\
& 1 &
\end{array}\right], \quad A_{1} \cong h^{-2}\left[\begin{array}{rrr}
0 & \\
1 & -2 & 1 \\
& 0 &
\end{array}\right], \quad A_{2} \cong h^{-2}\left[\begin{array}{rrr}
1 & \\
0 & -2 & 0 \\
& 1
\end{array}\right]
$$

Further, $f(t)=b(t)+g(t)$, where $g(t)$ is the restriction of $\mathbf{g}(x, y, t)$ to $\Omega_{h}$ and $b(t)=b_{1}(t)+b_{2}(t)$ comes from the boundary conditions, $b_{1}(t)$ having components $h^{-2} \mathbf{u}_{\Gamma}(x \pm h, y, t)$ on the gridpoints adjacent to the vertical boundaries and $b_{2}(t)$ components $h^{-2} \mathbf{u}_{\Gamma}(x, y \pm h, t)$ near the horizontal boundaries. For the source term $g(t)$, splittings $g(t)=g_{1}(t)+g_{2}(t)$ are considered with $g_{1}(t)=\theta g(t), g_{2}(t)=(1-\theta) g(t)$ and $\theta \in[0,1]$. We thus obtain a system (1.1), (1.2) with

$$
F_{j}(t, v)=A_{j} v+b_{j}(t)+g_{j}(t) \quad(j=1,2) .
$$

The errors of the LOD methods will be measured in the discrete $L_{2}$-norm

$$
\|w\|=\left[h^{2} \sum_{i, j=1}^{m}\left|w_{i j}\right|^{2}\right]^{1 / 2} \text { for } w=\left(w_{i j}\right) \in \mathbb{R}^{M} .
$$


The induced spectral norm for the space $L\left(\mathbb{R}^{M}\right)$ of $M \times M$ matrices is also denoted by $\|\cdot\|$. Along with the property that $A_{1}$ and $A_{2}$ commute, we shall often use the fact that these matrices are negative definite. This implies

$$
\left\|r\left(\tau A_{j}\right)\right\| \leq 1, \quad\left\|\left(I-\frac{1}{2} \tau A_{j}\right)^{-1}\right\| \leq 1, \quad \text { and } \quad\left\|\left(I-\frac{1}{2} \tau A_{j}\right)^{-1} \tau A_{j}\right\| \leq 2
$$

for all $\tau>0, j=1,2$, where $r(z)=\left(1-\frac{1}{2} z\right)^{-1}\left(1+\frac{1}{2} z\right)$ is the stability function of the implicit midpoint rule.

The fully discrete numerical solution $u_{n}$ will be compared with the exact PDE solution $\mathbf{u}$. This exact solution is assumed, throughout the paper, to be sufficiently often differentiable in time and space. The restriction of $\mathbf{u}(x, y, t)$ to $\Omega_{h}$ is denoted by $u_{h}(t)$, and it will be assumed that $u_{0}=u_{h}(0)$. The error due to space discretization is described by

$$
\alpha_{h}(t)=\dot{u}_{h}(t)-F\left(t, u_{h}(t)\right) .
$$

For our model problem this spatial error is $O\left(h^{2}\right)$.

The main objective of the present investigation is the temporal order of convergence $p$ appearing in the error bound

$$
\left\|u_{h}\left(t_{n}\right)-u_{n}\right\| \leq C \tau^{p}+D h^{2} \quad\left(\tau, h>0,0 \leq t_{n} \leq T\right)
$$

with constants $C, D$ independent of $\tau$ and $h$. Here, $\tau$ and $h$ are allowed to tend to zero simultaneously and independently of each other (unconditional convergence).

2.2. Some technical results. In the error bounds it will be required that the constants involved are not affected by $\tau$ and $h$. The symbol $O\left(\tau^{p} h^{q}\right)$ will be used to denote a scalar, or vector, whose absolute value, or $L_{2}$-norm, is bounded by $C \tau^{p} h^{q}$ for all possible $\tau, h>0$ with $C>0$ independent of $\tau$ and $h$. In particular, $O\left(\tau^{p}\right)$ thus stands for a term bounded by $C \tau^{p}$ for $\tau>0$ uniformly for $h>0$. Naturally, we always have $\tau \leq T$ and $h \leq 1$.

Let $\otimes$ denote the left Kronecker (or tensor) product of vectors in $\mathbb{R}^{m}$ and matrices in $L\left(\mathbf{R}^{m}\right)$, i.e.,

$$
v \otimes w=\left(w_{1} v^{\mathrm{T}}, w_{2} v^{\mathrm{T}}, \ldots, w_{m} v^{\mathrm{T}}\right)^{\mathrm{T}} \in \mathbb{R}^{M} \quad \text { for } v, w=\left(w_{i}\right) \in \mathbb{R}^{m}
$$

and $P \otimes S$ stands for the block matrix in $L\left(\mathbb{R}^{M}\right)$ with blocks $s_{i j} P \in L\left(\mathbb{R}^{m}\right)$ for $P, S=\left(s_{i j}\right) \in L\left(\mathbb{R}^{m}\right)$. Standard properties of such products can be found in [3, 7], for example. (We use the left Kronecker product rather than the more common right form, since it gives a more natural notation here.) A grid function with values $v\left(x_{i}\right) w\left(y_{j}\right)$ on $\Omega_{h}$ can be written as vector $v \otimes w$ with $v_{i}=v\left(x_{i}\right), w_{j}=w\left(y_{j}\right)$. It is easy to verify that

$$
\|v \otimes w\|=|v||w| \text { for } v, w \in \mathbb{R}^{m},
$$

where $|\cdot|$ is the one-dimensional discrete $L_{2}$-norm

$$
|v|=\left[h v^{\mathrm{T}} v\right]^{1 / 2} \text { for } v \in \mathbb{R}^{m} .
$$

Let $Q=h^{-2}$ tridiag $(-1,2,-1) \in L\left(\mathbb{R}^{m}\right)$ be the standard one-dimensional approximation for $-d^{2} / d x^{2}$. The matrices $A_{1}, A_{2}$ can be written as

$$
A_{1}=-Q \otimes I, \quad A_{2}=-I \otimes Q,
$$


$I$ being the identity matrix. The eigenvalues and eigenvectors of $Q$ are given by

$$
\begin{aligned}
& Q \phi_{i}=\lambda_{i} \phi_{i} \quad(1 \leq i \leq m), \quad \lambda_{i}=4 h^{-2} \sin ^{2}(i h \pi / 2), \\
& \phi_{i}=\sqrt{2}(\sin (i h \pi), \sin (2 i h \pi), \ldots, \sin (m i h \pi))^{\mathrm{T}} \in \mathbb{R}^{m} .
\end{aligned}
$$

Since $\left\{\phi_{1}, \phi_{2}, \ldots, \phi_{m}\right\}$ is an orthonormal set in $\mathbb{R}^{m}$ with respect to the inner product $h v^{\mathrm{T}} w$, we have for any $v \in \mathbb{R}^{m}$ the Fourier decomposition

$$
v=\sum_{i=1}^{m} \hat{v}_{i} \phi_{i}, \quad \hat{v}_{i}=h v^{\mathrm{T}} \phi_{i}(1 \leq i \leq m) \text { and }|v|^{2}=\sum_{i=1}^{m}\left|\hat{v}_{i}\right|^{2} .
$$

In the remainder of this section we consider a rational function $\psi$ such that

$$
\psi(0)=0 \text { and }|\psi(z)| \leq K \text { for all } z \leq 0 .
$$

Since $\tau Q$ is positive definite, it easily follows that for any $v \in \mathbb{R}^{m}$ a constant $C$ exists such that

$$
|\psi(-\tau Q) v| \leq C \text { for all } \tau, h>0
$$

(we can take $C=K|v|$; for this inequality the assumption $\psi(0)=0$ is irrelevant). If $v \in \mathbb{R}^{m}$ can be viewed as a smooth one-dimensional grid function, a better estimate is known for small $\tau$, also valid uniformly for $h>0$.

Lemma 2.1. Let $\chi \in C^{2}[0,1]$ and $v=\left(v_{i}\right) \in \mathbb{R}^{m}$ with $v_{i}=\chi(i h)$. Assume (2.8). Then, for any $\gamma<\frac{1}{4}$ there exists a $C_{\gamma}>0$ such that $|\psi(-\tau Q) v| \leq C_{\gamma} \tau^{\gamma}$ for all $\tau, h>0$.

This lemma can be proved as in the Appendix of [6], where $\psi(z)=$ $\left(1-\frac{1}{2} z\right)^{-1} z$ was considered. The constant $C_{\gamma}$ can be bounded in terms of $\gamma$ and upper bounds for $\left|z^{-\gamma} \psi(z)\right|(z \leq 0)$ and $|\chi(0)|,|\chi(1)|,\left|\chi^{\prime \prime}(x)\right|(0 \leq x \leq 1)$. The function $\chi$ thus may depend on $h$ as long as these upper bounds are valid uniformly in $h$.

Sharpness of the above result can be shown by considering the vector $e=$ $(1,1, \ldots, 1)^{\mathrm{T}} \in \mathbb{R}^{m}$.

Lemma 2.2. Let $\beta>0, \psi \neq 0$, and assume (2.8). There exists a $C>0$ such that

$$
|\psi(-\tau Q) e| \geq C \tau^{1 / 4} \text { for } \tau, h>0 \text { with } \tau / h^{2} \geq \beta .
$$

Also this lemma can be proved as in the Appendix of [6].

Note that if we were considering only a fixed meshwidth $h=h_{0}>0$, then for any $v \in \mathbb{R}^{m}$ it would hold that

$$
|\psi(-\tau Q) v| \leq C_{0} \tau \text { for } \tau>0, h=h_{0},
$$

where $C_{0}=|Q||v| \cdot \sup \left\{\left|z^{-1} \psi(z)\right|: z \leq 0\right\}$. However, since the spectral norm of $Q$ equals $\left|\lambda_{m}\right| \approx 4 h^{-2}$, such bounds are useless for small $h$. From the above estimates it is seen that bounds valid for all $h>0$ are possible, but at the cost of a lower power of $\tau$. This is essentially the cause for order reduction if $h \downarrow 0$ in time integration methods.

For vectors $w \in \mathbb{R}^{M}$, which can be regarded as grid functions on the twodimensional grid $\Omega_{h}$, we have

$$
w=\sum_{i, j=1}^{m} \hat{w}_{i j} \phi_{i} \otimes \phi_{j}, \quad\|w\|^{2}=\sum_{i, j=1}^{m}\left|\hat{w}_{i j}\right|^{2},
$$


with Fourier components $\hat{w}_{i j}=h^{2} w^{\mathrm{T}}\left[\phi_{i} \otimes \phi_{j}\right]$. Lemma 2.1 yields the following two-dimensional result.

Lemma 2.3. Let $\chi \in C^{2}\left([0,1]^{2}\right)$ and $w=\left(w_{i j}\right) \in \mathbb{R}^{M}$ with $w_{i j}=\chi(i h, j h)$. Assume (2.8). Then, for any $\gamma<\frac{1}{4}$ there is a $C_{\gamma}>0$ such that

$$
\left\|\psi\left(\tau A_{j}\right) w\right\| \leq C_{\gamma} \tau^{\gamma} \text { for all } \tau, h>0 \text { and } j=1,2 .
$$

Proof. Consider $j=1$ and the row-wise ordering $w=\left(w_{1}^{\mathrm{T}}, \ldots, w_{m}^{\mathrm{T}}\right)^{\mathrm{T}}, w_{j}=$ $\left(w_{1 j}, \ldots, w_{m j}\right)^{\mathrm{T}}$. We have (cf. [7])

$$
\psi\left(\tau A_{1}\right)=\psi(-\tau Q \otimes I)=\psi(-\tau Q) \otimes I,
$$

and consequently,

$$
\psi\left(\tau A_{1}\right) w=\left(\left(\psi(-\tau Q) w_{1}\right)^{\mathrm{T}}, \ldots,\left(\psi(-\tau Q) w_{m}\right)^{\mathrm{T}}\right)^{\mathrm{T}} .
$$

The bound for $\left\|\psi\left(\tau A_{1}\right) w\right\|$ now easily follows from Lemma 2.1.

For $j=2$ we can proceed in the same way by using a columnwise ordering for $w$.

\section{RECURSIONS FOR THE DISCRETIZATION ERRORS}

3.1. The basic scheme. In this section recursions for the global discretization errors $u_{h}\left(t_{n}\right)-u_{n}$ of the LOD schemes will be derived. As we shall see, the propagation of errors is governed by the matrix

$$
R=r\left(\tau A_{1}\right) r\left(\tau A_{2}\right) \text {. }
$$

Since, in view of (2.4), one has $\|R\| \leq 1$, stability is always ensured. In this section most attention will be given to the structure of the temporal discretization errors introduced in one single step of the integration process. These local discretization errors will be expressed in terms of derivatives of $u_{h}(t)$ and

$$
v_{h}(t)=F_{1}\left(t, u_{h}(t)\right)-F_{2}\left(t, u_{h}(t)\right) .
$$

Note that although $F_{1}$ and $F_{2}$ contain negative powers of $h$, the function $v_{h}$ and its derivatives are bounded uniformly for $h>0$, provided that the PDE solution $\mathbf{u}$ is sufficiently smooth (which will always be assumed).

Consider, along with (1.4), a perturbed scheme

$$
\begin{aligned}
& \tilde{u}_{n+1 / 2}=\tilde{u}_{n}+\tau F_{1}\left(t_{n}+\frac{1}{4} \tau, \frac{1}{2} \tilde{u}_{n}+\frac{1}{2} \tilde{u}_{n+1 / 2}+p_{n}\right)+q_{n}, \\
& \tilde{u}_{n+1}=\tilde{u}_{n+1 / 2}+\tau F_{2}\left(t_{n}+\frac{3}{4} \tau, \frac{1}{2} \tilde{u}_{n+1 / 2}+\frac{1}{2} \tilde{u}_{n+1}+p_{n+1 / 2}\right)+q_{n+1 / 2} .
\end{aligned}
$$

The perturbations $p_{k}, q_{k}$ may represent various error sources, for instance roundoff. Below, these perturbations will be used to derive suitable expressions for the local discretization errors.

Let $\varepsilon_{k}=\tilde{u}_{k}-u_{k}$ for $k=n, n+\frac{1}{2}$ and $n \geq 0$. By subtracting (1.4) from (3.3), we obtain

$$
\begin{aligned}
& \varepsilon_{n+1 / 2}=\varepsilon_{n}+\frac{1}{2} \tau A_{1}\left(\varepsilon_{n}+\varepsilon_{n+1 / 2}\right)+\tau A_{1} p_{n}+q_{n}, \\
& \varepsilon_{n+1}=\varepsilon_{n+1 / 2}+\frac{1}{2} \tau A_{2}\left(\varepsilon_{n+1 / 2}+\varepsilon_{n+1}\right)+\tau A_{2} p_{n+1 / 2}+q_{n+1 / 2} .
\end{aligned}
$$

Elimination of $\varepsilon_{n+1 / 2}$ leads to

$$
\varepsilon_{n+1}=R \varepsilon_{n}+\delta_{n} \quad(n=0,1,2, \ldots),
$$


where

$$
\begin{aligned}
\delta_{n}= & r\left(\tau A_{2}\right)\left(I-\frac{1}{2} \tau A_{1}\right)^{-1}\left[\tau A_{1} p_{n}+q_{n}\right] \\
& +\left(I-\frac{1}{2} \tau A_{2}\right)^{-1}\left[\tau A_{2} p_{n+1 / 2}+q_{n+1 / 2}\right] .
\end{aligned}
$$

If we put $\tilde{u}_{n}=u_{h}\left(t_{n}\right)$ for all $n \geq 0$, (3.4) gives a recursion for the global discretization errors. Evidently, $\delta_{n}$ is then a local discretization error. To obtain a suitable expression for these local errors, let $\tilde{u}_{n+1 / 2}=u_{h}\left(t_{n+1 / 2}\right)$ and

$$
\begin{aligned}
& p_{n}=-\frac{1}{2} u_{h}\left(t_{n}\right)+u_{h}\left(t_{n}+\frac{1}{4} \tau\right)-\frac{1}{2} u_{h}\left(t_{n}+\frac{1}{2} \tau\right), \\
& p_{n+1 / 2}=-\frac{1}{2} u_{h}\left(t_{n}+\frac{1}{2} \tau\right)+u_{h}\left(t_{n}+\frac{3}{4} \tau\right)-\frac{1}{2} u_{h}\left(t_{n}+\tau\right) .
\end{aligned}
$$

This choice is made so that all perturbations $p_{k}, q_{k}$ only depend on quantities like $u_{h}(t), F_{j}\left(t, u_{h}(t)\right)$, and time derivatives thereof, which are known to be bounded for $h \downarrow 0$.

By a Taylor expansion around $t=t_{n+1 / 2}$, and using the relation $\dot{u}_{h}=$ $F\left(t, u_{h}\right)+O\left(h^{2}\right)$, it follows with the above choice that

$$
\begin{aligned}
& p_{n}=-\frac{1}{32} \tau^{2} \ddot{u}_{h}\left(t_{n+1 / 2}\right)+O\left(\tau^{3}\right), \quad p_{n+1 / 2}=-\frac{1}{32} \tau^{2} \ddot{u}_{h}\left(t_{n+1 / 2}\right)+O\left(\tau^{3}\right), \\
& q_{n}=-\frac{1}{2} \tau v_{h}\left(t_{n+1 / 2}\right)+\frac{1}{8} \tau^{2} \dot{v}_{h}\left(t_{n+1 / 2}\right)+O\left(\tau^{3}\right)+O\left(\tau h^{2}\right), \\
& q_{n+1 / 2}=\frac{1}{2} \tau v_{h}\left(t_{n+1 / 2}\right)+\frac{1}{8} \tau^{2} \dot{v}_{h}\left(t_{n+1 / 2}\right)+O\left(\tau^{3}\right)+O\left(\tau h^{2}\right),
\end{aligned}
$$

with $v_{h}$ given by (3.2). Relation (3.5) can be written as

$$
\begin{aligned}
\delta_{n}=\left(I-\frac{1}{2} \tau A_{1}\right)^{-1}\left(I-\frac{1}{2} \tau A_{2}\right)^{-1}\left[\left(I+\frac{1}{2} \tau A_{2}\right)\right. & \left(\tau A_{1} p_{n}+q_{n}\right) \\
& \left.+\left(I-\frac{1}{2} \tau A_{1}\right)\left(\tau A_{2} p_{n+1 / 2}+q_{n+1 / 2}\right)\right] .
\end{aligned}
$$

Using the bounds (2.4), we obtain after some calculations

$$
\begin{aligned}
\delta_{n}=\left(I-\frac{1}{2} \tau A_{1}\right)^{-1}\left(I-\frac{1}{2} \tau A_{2}\right)^{-1}\left[\frac{1}{4} \tau^{2} \dot{v}_{h}\left(t_{n+1 / 2}\right)+\frac{1}{4} \tau^{2} A v_{h}\left(t_{n+1 / 2}\right)\right. & \\
& +\frac{1}{16} \tau^{3}\left(A_{2}-A_{1}\right) \dot{v}_{h}\left(t_{n+1 / 2}\right) \\
& +O\left(\tau^{3}\right)+O\left(\tau h^{2}\right) .
\end{aligned}
$$

So, the global discretization errors $\varepsilon_{n}=u_{h}\left(t_{n}\right)-u_{n}$ of the basic LOD scheme (1.5) satisfy the recursion (3.4) with local errors $\delta_{n}$ given by (3.6). In $\S \S 4$ and 5 this will be used to derive error bounds.

3.2. The alternating schemes. For the alternating schemes (1.6), (1.7) recursions for the global errors $\varepsilon_{n}=u_{h}\left(t_{n}\right)-u_{n}$ are easily obtained from the previous results. Let $\delta_{n}$ be the local errors, given by (3.6), of the basic scheme $u_{n+1}=$ $\Phi_{1,2}\left(u_{n}\right)$. The scheme $u_{n+1}=\Phi_{2,1}\left(u_{n}\right)$ has local errors

$$
\begin{aligned}
\delta_{n}^{\prime}=\left(I-\frac{1}{2} \tau A_{1}\right)^{-1}\left(I-\frac{1}{2} \tau A_{2}\right)^{-1}[ & -\frac{1}{4} \tau^{2} \dot{v}_{h}\left(t_{n+1 / 2}\right)+\frac{1}{4} \tau^{2} A v_{h}\left(t_{n+1 / 2}\right) \\
& +\frac{1}{16} \tau^{3}\left(A_{2}-A_{1}\right) \dot{v}_{h}\left(t_{n+1 / 2}\right) \\
& +O\left(\tau^{3}\right)+O\left(\tau h^{2}\right) .
\end{aligned}
$$

This follows directly from (3.2), (3.6) by interchanging the indices 1,2 .

For the scheme (1.6) we thus have

$$
\varepsilon_{n+1}=R \varepsilon_{n}+\delta_{n}, \quad \varepsilon_{n+2}=R \varepsilon_{n+1}+\delta_{n+1}^{\prime} \quad(n=0,2,4, \ldots) .
$$


Taking the two steps together, it follows that

$$
\varepsilon_{n+2}=R^{2} \varepsilon_{n}+\rho_{n}, \quad \rho_{n}=R \delta_{n}+\delta_{n+1}^{\prime} \quad(n=0,2,4, \ldots) .
$$

Likewise, we get for the parallel alternating scheme (1.7) the error recursion

$$
\varepsilon_{n+1}=R \varepsilon_{n}+\sigma_{n}, \quad \sigma_{n}=\frac{1}{2} \delta_{n}+\frac{1}{2} \delta_{n}^{\prime} \quad(n=0,1,2, \ldots) .
$$

Its local error $\sigma_{n}$ can be written as

$$
\begin{aligned}
\sigma_{n}=\left(I-\frac{1}{2} \tau A_{1}\right)^{-1}\left(I-\frac{1}{2} \tau A_{2}\right)^{-1} & {\left[\frac{1}{16} \tau^{3}\left(A_{2}-A_{1}\right) \dot{v}_{h}\left(t_{n+1 / 2}\right)\right.} \\
& \left.-\frac{1}{32} \tau^{3} A \ddot{u}_{h}\left(t_{n+1 / 2}\right)\right]+O\left(\tau^{3}\right)+O\left(\tau h^{2}\right) .
\end{aligned}
$$

\section{LOCAL ERROR BOUNDS}

4.1. The basic scheme. For a time integration method consistent of order $p$ one would expect local discretization errors to behave like $O\left(\tau^{p+1}\right)+O\left(\tau h^{2}\right)$, with the $\tau h^{2}$ contribution coming from spatial errors.

Consider the local error $\delta_{n}$, given by (3.6), of the basic scheme (1.5), which has order 1 in the classical ODE sense. It is easily seen, by using (2.4), that $\left\|\delta_{n}\right\|=O\left(\tau^{2}\right)+O\left(\tau h^{2}\right)$, provided that

$$
\left\|A v_{h}(t)\right\|=O(1)
$$

uniformly in $h$. This condition, however, will not hold in general, because of the fact that even if the PDE solution $\mathbf{u}$ is very smooth, $v_{h}(t)$ (which then also is a smooth grid function) need not be zero near the boundaries $\Gamma$. For smooth $\mathbf{u},(4.1)$ is equivalent to the compatibility condition

$$
\left(\frac{\partial^{2}}{\partial x^{2}}-\frac{\partial^{2}}{\partial y^{2}}\right) \mathbf{u}(x, y, t)+(2 \theta-1) \mathbf{g}(x, y, t)=0 \text { on } \Gamma,
$$

and this will only be satisfied in exceptional cases, e.g., if $\mathbf{g} \equiv 0, \mathbf{u}_{\Gamma} \equiv 0$.

In the following it will not be assumed that (4.1) holds. It is then still possible to obtain upper bounds for the local errors uniformly for $h>0$, but with a reduced order of consistency.

Lemma 4.1. For any $\gamma<\frac{1}{4}$ there are constants $C_{\gamma}, D>0$ such that

$$
\left\|\delta_{n}\right\| \leq C_{\gamma} \tau^{1+\gamma}+D \tau h^{2} \text { for all } \tau, h>0,0 \leq t_{n} \leq T .
$$

Proof. From (3.6) and the bounds (2.4) it follows that

$$
\delta_{n}=-\frac{1}{4} \tau^{2}\left(I-\frac{1}{2} \tau A_{1}\right)^{-1}\left(I-\frac{1}{2} \tau A_{2}\right)^{-1} A v_{h}\left(t_{n+1 / 2}\right)+O\left(\tau^{2}\right)+O\left(\tau h^{2}\right)
$$

and

$$
\left\|\delta_{n}\right\| \leq \frac{1}{4} \tau^{2} \sum_{j=1}^{2}\left\|\left(I-\frac{1}{2} A_{j}\right)^{-1} A_{j} v_{h}\left(t_{n+1 / 2}\right)\right\|+O\left(\tau^{2}\right)+O\left(\tau h^{2}\right) .
$$

Since $v_{h}(t)$ is a smooth grid function, provided $\mathbf{u}$ is smooth, the result now follows from Lemma 2.3.

In the above estimate we have obtained a temporal order of consistency $\approx \frac{1}{4}$ only. To show that this result is sharp, we will consider problem (2.1) with a suitably chosen simple solution. This solution can be taken stationary. 
Example 4.2. Consider a distribution of the source term with $\theta=0$; other natural choices $\theta=\frac{1}{2}$ or $\theta=1$ can be treated similarly. Let

$$
\mathbf{u}(x, y, t)=\frac{1}{2} x(1-x) \sin (\pi y) .
$$

Then $g_{2}(t)=g(t)$ is the restriction of $-\Delta \mathbf{u}(x, y, t)$ to $\Omega_{h}$, and $b(t)=0$, since u satisfies homogeneous boundary conditions. It follows by some simple calculations that

$$
v_{h}(t)=-2 e \otimes \phi_{1}+O\left(h^{2}\right),
$$

where $e=(1,1, \ldots, 1)^{\mathrm{T}} \in \mathbb{R}^{m}$ and $\phi_{1}$ is the first eigenvector of $Q$ (cf. $\S 2.2$ ).

To simplify the situation further, we neglect in the following the spatial errors $O\left(h^{2}\right)$; it is only the temporal order which is of interest here. So, we consider the local error

$$
\delta_{n}=\frac{1}{2} \tau^{2}\left(I-\frac{1}{2} \tau A_{1}\right)^{-1}\left(I-\frac{1}{2} \tau A_{2}\right)^{-1} A\left[e \otimes \phi_{1}\right] .
$$

This can be written as

$$
\begin{aligned}
\delta_{n}= & -\frac{1}{2} \tau^{2}\left[\left(I+\frac{1}{2} \tau Q\right)^{-1} Q e \otimes\left(I+\frac{1}{2} \tau Q\right)^{-1} \phi_{1}\right] \\
& -\frac{1}{2} \tau^{2}\left[\left(I+\frac{1}{2} \tau Q\right)^{-1} e \otimes\left(I+\frac{1}{2} \tau Q\right)^{-1} Q \phi_{1}\right] .
\end{aligned}
$$

The second term on the right-hand side is of $O\left(\tau^{2}\right)$ since $Q \phi_{1}=\lambda_{1} \phi_{1}=O(1)$ and $\left(I+\frac{1}{2} \tau Q\right)^{-1}$ is also bounded for all $h>0$. Using Lemma 2.2, we can see that the norm of the first term is bounded from below by $C \tau^{5 / 4}, C>0$, provided that $\tau / h^{2} \neq o(1)$, and consequently, such a lower bound also holds for $\left\|\delta_{n}\right\|$. Hence, the order result in Lemma 4.1 cannot be essentially improved if both $\tau$ and $h$ tend to zero and $\tau / h^{2} \geq \beta>0$.

4.2. The sequentially alternating scheme. Consider the local error $\rho_{n}$ in (3.9) of scheme (1.6). By some calculations it can be seen that the temporal order of consistency is 2 , provided $\mathbf{u}$ satisfies certain compatibility conditions, or if $h \geq h_{0}>0$. So, it seems that compared to the basic scheme (1.5), one order is gained. Below it will be shown, however, that this gain is lost in general as $h \downarrow 0$.

Observe that, since $\|R\| \leq 1$,

$$
\left\|\rho_{n}\right\| \leq\left\|\delta_{n}\right\|+\left\|\delta_{n+1}^{\prime}\right\| \text {. }
$$

The bound of Lemma 4.1 for $\left\|\delta_{n}\right\|$ holds for $\left\|\delta_{n+1}^{\prime}\right\|$ as well. Therefore, the following result follows directly.

Lemma 4.3. For any $\gamma<\frac{1}{4}$ there are $C_{\gamma}, D>0$ such that

$$
\left\|\rho_{n}\right\| \leq C_{\gamma} \tau^{1+\gamma}+D \tau h^{2} \text { for all } \tau, h>0,0 \leq t_{n} \leq T .
$$

To prove sharpness, we consider again a simple stationary problem.

Example 4.4. Let, as in Example 4.2, $\mathbf{u}$ be given by (4.3) and $\theta=0$. Omitting the spatial errors, it follows from (3.7), (4.4), and (4.5) that $\delta_{n+1}^{\prime}=-\delta_{n}$ and

$$
\rho_{n}=(R-I) \delta_{n}=\frac{1}{2} \tau^{3}\left(I-\frac{1}{2} \tau A_{1}\right)^{-2}\left(I-\frac{1}{2} \tau A_{2}\right)^{-2} A^{2}\left[e \otimes \phi_{1}\right] .
$$


Hence,

$$
\begin{aligned}
\rho_{n}= & \frac{1}{2} \tau^{3}\left[\left(I+\frac{1}{2} \tau Q\right)^{-2} Q^{2} e \otimes\left(I+\frac{1}{2} \tau Q\right)^{-2} \phi_{1}\right] \\
& +\tau^{3}\left[\left(I+\frac{1}{2} \tau Q\right)^{-2} Q e \otimes\left(I+\frac{1}{2} \tau Q\right)^{-2} Q \phi_{1}\right] \\
& +\frac{1}{2} \tau^{3}\left[\left(I+\frac{1}{2} \tau Q\right)^{-2} e \otimes\left(I+\frac{1}{2} \tau Q\right)^{-2} Q^{2} \phi_{1}\right] .
\end{aligned}
$$

Now, let $\tau, h \downarrow 0$ and $\tau / h^{2} \geq \beta>0$. Using Lemma 2.2, we see that the norm of the first term on the right-hand side is bounded from below by $C \tau^{5 / 4}, C>0$. The second and third terms are $O\left(\tau^{9 / 4}\right)$ and $O\left(\tau^{3}\right)$, respectively. Hence, for $\left\|\rho_{n}\right\|$ we have a lower bound $C^{\prime} \tau^{5 / 4}$ with a constant $C^{\prime}>0$.

4.3. The parallel alternating scheme. The local error $\sigma_{n}$ of the parallel alternating scheme (1.7) is given by (3.11). In the same way as in Lemma 4.1 it can be shown that the following local bound is valid.

Lemma 4.5. For any $\gamma<\frac{1}{4}$ there are $C_{\gamma}, D>0$ such that

$$
\left\|\sigma_{n}\right\| \leq C_{\gamma} \tau^{2+\gamma}+D \tau h^{2} \text { for all } \tau, h>0,0 \leq t_{n} \leq T .
$$

Compared to the other two schemes, one order of $\tau$ has been gained. Note, however, that there still is an order reduction, since for fixed ODE's, scheme (1.7) is consistent with order 2. To show that the above bound is sharp when $h \downarrow 0$ we consider again a simple example.

Example 4.6. Suppose $\theta=0$. As before, other choices for $\theta$ can be treated similarly. Let

$$
\mathbf{u}(x, y, t)=\frac{1}{2} t x(1-x) \sin (\pi y) .
$$

Note that here a nonstationary solution is considered; for stationary $u$ the scheme $(1.7)$ is exact in time. For the above problem we have $\ddot{u}_{h}(t)=0$ and

$$
\dot{v}_{h}(t)=-2 e \otimes \phi_{1}+O\left(h^{2}\right) .
$$

Hence, omitting the spatial errors,

$$
\sigma_{n}=-\frac{1}{8} \tau^{3}\left(I-\frac{1}{2} \tau A_{1}\right)^{-1}\left(I-\frac{1}{2} \tau A_{2}\right)^{-1}\left(A_{2}-A_{1}\right)\left[e \otimes \phi_{1}\right],
$$

which can be written as

$$
\begin{aligned}
\sigma_{n}= & -\frac{1}{8} \tau^{3}\left[\left(I+\frac{1}{2} \tau Q\right)^{-1} Q e \otimes\left(I+\frac{1}{2} \tau Q\right)^{-1} \phi_{1}\right] \\
& +\frac{1}{8} \tau^{3}\left[\left(I+\frac{1}{2} \tau Q\right)^{-1} e \otimes\left(I+\frac{1}{2} \tau Q\right)^{-1} Q \phi_{1}\right] .
\end{aligned}
$$

Comparing this with formula (4.6), it now follows that $\left\|\sigma_{n}\right\| \geq C \tau^{9 / 4}$ with $C>0$, if $\tau, h \downarrow 0$ and $\tau / h^{2} \geq \beta>0$.

In the examples treated in this section the order reduction has been caused by the presence of a source term $\mathbf{g}$. The same effect would have been obtained if we had taken $\mathbf{g} \equiv 0$ but boundary values $u_{\Gamma}$ varying in time, for instance with

$$
\mathbf{u}(x, y, t)=\exp \left(-\frac{5}{4} \pi^{2} t\right) \sin (\pi x) \sin (\pi y / 2) .
$$

\section{GLOBAL ERROR BOUNDS}

5.1. Global order reduction. Consider an error recursion in $\mathbb{R}^{M}$ of the form

$$
e_{n+1}=S e_{n}+d_{n} \quad(n=0,1, \ldots, N-1), \quad e_{0}=0,
$$


where $N=\lfloor T / \tau\rfloor$ and $S \in L\left(\mathbb{R}^{M}\right),\|S\| \leq 1$. The vectors $e_{n}$ and $d_{n}$ stand for global and local errors, respectively. If $\left\|\bar{d}_{n}\right\|=O\left(\tau^{1+\gamma}\right)+O\left(\tau h^{2}\right)$ uniformly for $n \geq 0$, we obtain in the standard way the global result $\left\|e_{n}\right\|=O\left(\tau^{\gamma}\right)+O\left(h^{2}\right)$, i.e., convergence with temporal order $\gamma$. In many cases, however, it is possible to improve this global result by taking into account certain cancellation effects, giving a temporal order of convergence which is larger than the temporal order of consistency. Consequently, the order reduction in the global errors is often less prominent than in the local errors; it may even be absent. In this section this will be shown to be the case for the schemes (1.5) and (1.7) (not for scheme (1.6), however). Similar results for Runge-Kutta methods can be found in [2, 12], for example, and for an ADI method in [6]. 0 ,

Let $\alpha>0$, and consider the following two statements with constants $C, C^{\prime}>$

$$
\begin{aligned}
& \left\|e_{n}\right\| \leq C \tau^{\alpha} \quad(0 \leq n \leq N), \\
& d_{n}=(I-S) \xi_{n}+\eta_{n}(0 \leq n \leq N-1) \text { with } \xi_{n}, \eta_{n} \in \mathbb{R}^{M} \text { such that } \\
& \left\|\xi_{n}\right\| \leq C^{\prime} \tau^{\alpha},\left\|\eta_{n}\right\| \leq C^{\prime} \tau^{1+\alpha}(0 \leq n \leq N-1) \text { and } \\
& \left\|\xi_{n}-\xi_{n-1}\right\| \leq C^{\prime} \tau^{1+\alpha}(1 \leq n \leq N-1) .
\end{aligned}
$$

Lemma 5.1. Assume (5.3). Then (5.2) holds with $C$ depending only on $C^{\prime}$ and $T$.

Proof (cf. also [5]). Instead of directly bounding the local errors $d_{n}$, we first expand the recursion (5.1), giving

$$
\begin{aligned}
e_{n}= & d_{n-1}+S d_{n-2}+\cdots+S^{n-1} d_{0} \\
= & \xi_{n-1}-S\left(\xi_{n-1}-\xi_{n-2}\right)-\cdots-S^{n-1}\left(\xi_{1}-\xi_{0}\right)-S^{n} \xi_{0} \\
& +\eta_{n-1}+S \eta_{n-2}+\cdots+S^{n-1} \eta_{0} .
\end{aligned}
$$

Hence, by bounding the terms in this last sum,

$$
\left\|e_{n}\right\| \leq 2 C^{\prime} \tau^{\alpha}+2 n C^{\prime} \tau^{1+\alpha} \leq 2(1+T) C^{\prime} \tau^{\alpha} \quad \text { for } n \tau \leq T .
$$

In case the local errors are constant, the reverse implication also holds.

Lemma 5.2. Assume (5.2) and $d_{n}=d_{0}(0 \leq n \leq N-1)$. Then there are $\xi_{n}=\xi_{0}, \eta_{n}=\eta_{0}$ such that (5.3) holds, with $C^{\prime}$ depending only on $C$ and $T$. Proof. Let $\lambda=(1+\tau)^{-1}$, so that $\|\lambda S\|<1$. Consider a sequence $\left\{v_{n}\right\}$ defined by

Then, for $w_{n}=v_{n}-e_{n}$, we have

$$
v_{n+1}=\lambda S v_{n}+d_{0} \quad(n \geq 0), \quad v_{0}=0 .
$$

$$
w_{n+1}=\lambda S w_{n}-(1-\lambda) S e_{n} \quad(n \geq 0), \quad w_{0}=0,
$$

and hence

$$
\left\|w_{n+1}\right\| \leq \lambda\left\|w_{n}\right\|+(1-\lambda) C \tau^{\alpha} \quad(n \geq 0), \quad\left\|w_{0}\right\|=0 .
$$

This leads in a standard way to the global result

$$
\left\|w_{n}\right\| \leq\left(1+\lambda+\cdots+\lambda^{n-1}\right)(1-\lambda) C \tau^{\alpha}=\left(1-\lambda^{n}\right) C \tau^{\alpha} \leq C \tau^{\alpha} .
$$

Thus, we obtain

$$
\left\|v_{n}\right\| \leq\left\|e_{n}\right\|+\left\|w_{n}\right\| \leq 2 C \tau^{\alpha} \quad(n \geq 0) .
$$


On the other hand, from the recursion for the $v_{n}$ it follows that

$$
v_{N}=\left(I+\lambda S+\cdots+\lambda^{N-1} S^{N-1}\right) d_{0}=\left(I-\lambda^{N} S^{N}\right)(I-\lambda S)^{-1} d_{0} .
$$

Therefore,

$$
\left\|(I-\lambda S)^{-1} d_{0}\right\| \leq\left(1-\lambda^{N}\right)^{-1} 2 C \tau^{\alpha} \leq C^{\prime} \tau^{\alpha}
$$

with $C^{\prime}=2 C(1+T) / T$.

Now take

$$
\xi_{0}=(I-\lambda S)^{-1} d_{0}, \quad \eta_{0}=(1-\lambda) S \xi_{0} .
$$

Then it is easily seen that $d_{0}=(I-S) \xi_{0}+\eta_{0}$, and from the above it follows that $\left\|\xi_{0}\right\| \leq C^{\prime} \tau^{\alpha},\left\|\eta_{0}\right\| \leq C^{\prime} \tau^{1+\alpha}$.

It should be noted that if $S=I+O(\tau)$, then (5.3) is simply equivalent to $\left\|d_{n}\right\|=O\left(\tau^{1+\alpha}\right)$, and (5.2) would follow in the standard way. The decomposition (5.3) can be useful if $S$ is bounded away from $I$, as will be the case if $S=R=r\left(\tau A_{1}\right) r\left(\tau A_{2}\right)$ when $h \downarrow 0$. Then (5.3) merely implies $\left\|d_{n}\right\|=O\left(\tau^{\alpha}\right)$.

Remark 5.3. For simplicity, only temporal errors were considered in the above, but it easily follows that

$$
\left\|e_{n}\right\| \leq C \tau^{\alpha}+D h^{2} \quad(0 \leq n \leq N)
$$

if (5.3) is satisfied with $\left\|\xi_{n}\right\| \leq C^{\prime} \tau^{\alpha},\left\|\xi_{n}-\xi_{n-1}\right\| \leq C^{\prime} \tau^{1+\alpha}$, and $\left\|\eta_{n}\right\| \leq$ $C^{\prime} \tau^{1+\alpha}+D^{\prime} \tau h^{2}$.

5.2. The basic scheme. Scheme (1.5) is convergent with temporal order 1 in the classical ODE sense. The following theorem shows that this remains valid as $h \downarrow 0$. So, in the transition from local to global error the order reduction disappears.

Theorem 5.4. Consider (1.5) and (2.1). There are constants $C, D>0$, depending only on $T$ and the smoothness of $\mathbf{u}$, such that

$$
\left\|u_{h}\left(t_{n}\right)-u_{n}\right\| \leq C \tau+D h^{2} \text { for all } \tau, h>0,0 \leq t_{n} \leq T \text {. }
$$

Proof. Let $\xi_{n}=\frac{1}{4} \tau v_{h}\left(t_{n+1 / 2}\right)$ and $\eta_{n}=\delta_{n}-(I-R) \xi_{n}$. Smoothness of $\mathbf{u}$ implies $\left\|\xi_{n}\right\|=O(\tau),\left\|\xi_{n}-\xi_{n-1}\right\|=O\left(\tau^{2}\right)$. By observing that

$$
I-R=-\tau\left(I-\frac{1}{2} \tau A_{2}\right)^{-1}\left(I-\frac{1}{2} \tau A_{2}\right)^{-1} A,
$$

it can be seen from (2.4) that $\left\|\eta_{n}\right\|=O\left(\tau^{2}\right)+O\left(\tau h^{2}\right)$. The proof follows from Lemma 5.1 and Remark 5.3.

5.3. The sequentially alternating scheme. As we saw in $\S 4.2$, modifying (1.5) into (1.6) did not help to improve the temporal order of consistency. Here it will be shown that this modification even has a negative effect on the order of convergence in time.

Example 5.5. Consider the problem of Examples 4.2, 4.4 with $\tau=h$. Omitting the space errors, we have for this stationary example the error recursion

$$
\varepsilon_{n+2}=R^{2} \varepsilon_{n}+\frac{1}{2} \tau(R-I)^{2} e \otimes \phi_{1} \quad(n=0,2,4, \ldots)
$$

(cf. (3.9), (4.7), and (5.4)). According to Lemmas 5.1 and 5.2 there will be convergence with temporal order $\alpha$ if and only if there are $\xi, \eta \in \mathbb{R}^{M}$ such that

$$
\|\xi\|=O\left(\tau^{\alpha}\right), \quad\|\eta\|=O\left(\tau^{1+\alpha}\right),
$$




$$
\frac{1}{2} \tau(R-I)^{2} e \otimes \phi_{1}=\left(R^{2}-I\right) \xi+\eta .
$$

It will be shown that this necessarily implies $\alpha \leq \frac{1}{2}$.

We consider the Fourier expansions

$$
\xi=\sum_{i, j=1}^{m} \hat{\xi}_{i j} \phi_{i} \otimes \phi_{j}, \quad \eta=\sum_{i, j=1}^{m} \hat{\eta}_{i j} \phi_{i} \otimes \phi_{j}, \quad \text { and } \quad e=\sum_{i=1}^{m} \hat{e}_{i} \phi_{i} .
$$

Let further $\lambda_{1}, \ldots, \lambda_{m}$ be the eigenvalues of $Q$ and $r_{i}=r\left(-\tau \lambda_{i}\right)$ (cf. §2.2). By observing that $R \phi_{i} \otimes \phi_{j}=r_{i} r_{j} \phi_{i} \otimes \phi_{j}$, it follows from (5.6b) that

$$
0=\left(r_{i}^{2} r_{j}^{2}-1\right) \hat{\xi}_{i j}+\hat{\eta}_{i j} \text { for } 1 \leq i \leq m, 2 \leq j \leq m .
$$

This is fulfilled by taking $\hat{\xi}_{i j}=\hat{\eta}_{i j}=0$ for $j \geq 2$; any other choice would lead only to larger norms $\|\xi\|$ and $\|\eta\|$. With $j=1$ we obtain from (5.6b)

$$
\frac{1}{2} \tau\left(r_{1} r_{i}-1\right)^{2} \hat{e}_{i}=\left(r_{1} r_{i}^{2}-1\right) \hat{\xi}_{1 i}+\hat{\eta}_{1 i} \text { for } 1 \leq i \leq m .
$$

To simplify this relation, define

$$
\hat{\xi}_{i}=2\left(\frac{r_{i}-1}{r_{1} r_{i}-1}\right)^{2}\left(\frac{r_{1}^{2} r_{i}^{2}-1}{r_{i}^{2}-1}\right) \hat{\xi}_{i 1}, \quad \hat{\eta}_{i}=2\left(\frac{r_{i}-1}{r_{1} r_{i}-1}\right)^{2} \hat{\eta}_{i 1} .
$$

Since $r_{1}=1+O(\tau)$, it now follows that (5.6) reduces to

$$
\left[\sum_{i=1}^{m}\left|\hat{\xi}_{i}\right|^{2}\right]^{1 / 2}=O\left(\tau^{\alpha}\right), \quad\left[\sum_{i=1}^{m}\left|\hat{\eta}_{i}\right|^{2}\right]^{1 / 2}=O\left(\tau^{1+\alpha}\right),
$$

$$
\tau\left(r_{i}-1\right)^{2} \hat{e}_{i}=\left(r_{i}^{2}-1\right) \hat{\xi}_{i}+\hat{\eta}_{i} \text { for } 1 \leq i \leq m .
$$

In the following we will use the notation $f(x) \sim g(x)(x \downarrow 0)$ for real functions $f, g$ if there are $C_{0}, C_{1}, H>0$ such that $C_{0} g(x) \leq f(x) \leq C_{1} g(x)$ for $0<x<H$. From (2.7) it is easily seen that $\lambda_{i} \sim i^{2}(h \downarrow 0)$ uniformly for $1 \leq i \leq m$.

Now, consider the indices $i \geq \frac{1}{2} m$. Since we assumed $\tau=h$, we have, uniformly for these large indices, $r_{i}-1 \sim-1$ and $r_{i}^{2}-1 \sim-\left(r_{i}+1\right) \sim-\tau$ $(\tau \downarrow 0)$. Hence, $(5.7 b)$ implies

$$
\tau^{2} \sum_{i \geq m / 2}\left|\hat{e}_{i}\right|^{2} \sim \sum_{i \geq m / 2}\left|\hat{\eta}_{i}-\tau \hat{\xi}_{i}\right|^{2}
$$

From the proof of Lemma 2, p. 99 in [6] with $\gamma=0$ it can be seen that

$$
\hat{e}_{i}= \begin{cases}0 & \text { for } i \text { even, } \\ h \sqrt{2} \operatorname{cotan}(i h \pi / 2) & \text { for } i \text { odd },\end{cases}
$$

and that for $\tau=h \downarrow 0$,

$$
\sum_{i \geq m / 2}\left|\hat{e}_{i}\right|^{2} \sim h^{2} \sum_{x_{i} \geq 1 / 2} \operatorname{cotan}^{2}\left(x_{i} \pi / 2\right) \sim h \int_{1 / 2}^{1} \operatorname{cotan}^{2}(x \pi / 2) d x \sim h=\tau .
$$

On the other hand, we have in view of $(5.7 a)$

$$
\sum_{i \geq m / 2}\left|\hat{\eta}_{i}-\tau \xi_{i}\right|^{2}=O\left(\tau^{2+2 \alpha}\right) .
$$


Hence, (5.8) implies $\tau^{3}=O\left(\tau^{2+2 \alpha}\right)$, and thus we must have $\alpha \leq \frac{1}{2}$.

Note that from the local error bound $\left\|\rho_{n}\right\|=O\left(\tau^{1+\gamma}\right)+O\left(\tau h^{2}\right)$ of Lemma 4.3 with $\gamma \approx \frac{1}{4}$, it directly follows that the temporal order of convergence is always at least $\frac{1}{4}$, approximately. With the above example we now know that this order result is nearly optimal. (The question whether the order is $\frac{1}{4}$ or $\frac{1}{2}$, or in between, is not so relevant since the convergence behavior is very disappointing anyway.)

So, we have the surprising result that scheme (1.6), which has a higher order in the classical ODE sense than (1.5), has a lower order of convergence in time when $\tau$ and $h$ tend to 0 simultaneously. To give an illustration of this, we present some numerical results for (2.1) with $T=2$ and

$$
\mathbf{u}(x, y, t)=x(1-x) y(1-y)(16+y) .
$$

This solution is chosen so that no space errors are present and its magnitude is near 1 . The source term $\mathbf{g}$ in (2.1) equals $-\Delta \mathbf{u}$, and we take $g_{1}=g_{2}=\frac{1}{2} g$ (i.e., $\theta=\frac{1}{2}$ ). Tables 5.1 and 5.2 nicely illustrate the theory. On fixed space grids, where we are in the standard ODE situation, the sequentially alternating scheme (1.6) becomes more accurate than the basic scheme (1.5) for decreasing $\tau$, but if both $\tau$ and $h$ tend to 0 , scheme (1.5) is the better one.

TABLE 5.1

Global errors $\left(L_{2}\right.$-norms) for $(1.5),(1.6)$ on a fixed space grid $h=\frac{1}{5}$

\begin{tabular}{|c|c|c|c|c|c|}
\hline$\tau^{-1}$ & 10 & 20 & 40 & 80 & 160 \\
\hline$(1.5)$ & $0.47 \mathrm{E}-1$ & $0.23 \mathrm{E}-1$ & $0.12 \mathrm{E}-1$ & $0.59 \mathrm{E}-2$ & $0.29 \mathrm{E}-2$ \\
$(1.6)$ & $0.69 \mathrm{E}-1$ & $0.32 \mathrm{E}-1$ & $0.10 \mathrm{E}-1$ & $0.27 \mathrm{E}-2$ & $0.68 \mathrm{E}-3$ \\
\hline
\end{tabular}

TABLE 5.2

Global errors $\left(L_{2}\right.$-norm) for $(1.5),(1.6)$ with $h=2 \tau$

\begin{tabular}{|c|c|c|c|c|c|}
\hline$\tau^{-1}$ & 10 & 20 & 40 & 80 & 160 \\
\hline$(1.5)$ & $0.47 \mathrm{E}-1$ & $0.34 \mathrm{E}-1$ & $0.20 \mathrm{E}-1$ & $0.10 \mathrm{E}-1$ & $0.53 \mathrm{E}-2$ \\
$(1.6)$ & $0.69 \mathrm{E}-1$ & $0.63 \mathrm{E}-1$ & $0.45 \mathrm{E}-1$ & $0.31 \mathrm{E}-1$ & $0.22 \mathrm{E}-1$ \\
\hline
\end{tabular}

TABLE 5.3

Global errors (max-norm) for (1.5), (1.6) with $h=2 \tau$

\begin{tabular}{|c|c|c|c|c|c|}
\hline$\tau^{-1}$ & 10 & 20 & 40 & 80 & 160 \\
\hline$(1.5)$ & $0.74 \mathrm{E}-1$ & $0.73 \mathrm{E}-1$ & $0.46 \mathrm{E}-1$ & $0.25 \mathrm{E}-1$ & $0.13 \mathrm{E}-1$ \\
$(1.6)$ & 0.11 & 0.14 & 0.14 & 0.14 & 0.14 \\
\hline
\end{tabular}


Although the theory has been formulated for the discrete $L_{2}$-norm, it is illuminating to consider the errors of Table 5.2 also in the maximum norm $\|v\|_{\infty}=\max \left|v_{i j}\right|, v=\left(v_{i j}\right) \in \mathbb{R}^{M}$. In this norm the behavior of the sequentially alternating scheme is even worse: it appears that there is no convergence at all.

The fact that in the above tables the asymptotic behavior does not show up for the larger $\tau$ values is probably caused by damping effects of terms $\left(I-\frac{1}{2} \tau A_{j}\right)^{-1}$, which will be stronger the larger $\tau$ is. In the error estimates we have merely used the bound $\left\|\left(I-\frac{1}{2} \tau A_{j}\right)^{-1}\right\| \leq 1$ for all $\tau>0$.

5.4. The parallel alternating scheme. As we saw in $\S 4$, the local errors of the parallel alternating scheme (1.7) also suffer from order reduction, though not as much as for scheme (1.6). Moreover, the following theorem shows that this reduction will be annihilated for the global errors of (1.7).

Theorem 5.6. Consider (1.7) and (2.1). There are constants $C, D>0$, depending only on $T$ and the smoothness of $\mathbf{u}$, such that

$$
\left\|u_{h}\left(t_{n}\right)-u_{n}\right\| \leq C \tau^{2}+D h^{2} \text { for all } \tau, h>0,0 \leq t_{n} \leq T .
$$

Proof. Let

$$
\begin{aligned}
& \xi_{n}=-\frac{1}{16} \tau^{2} A^{-1}\left(A_{2}-A_{1}\right) \dot{v}_{h}\left(t_{n+1 / 2}\right)+\frac{1}{32} \tau^{2} \ddot{u}_{h}\left(t_{n+1 / 2}\right), \\
& \eta_{n}=\sigma_{n}-(I-R) \xi_{n} .
\end{aligned}
$$

Note that $A^{-1}\left(A_{2}-A_{1}\right)$ is symmetric with eigenvalues $\left(\lambda_{i}+\lambda_{j}\right)^{-1}\left(\lambda_{i}-\lambda_{j}\right)$ bounded by 1 in modulus, and hence the norm of this matrix is $\leq 1$. Smoothness of $\mathbf{u}$ thus implies $\left\|\xi_{n}\right\|=O\left(\tau^{2}\right)$ and $\left\|\xi_{n}-\xi_{n-1}\right\|=O\left(\tau^{3}\right)$. Further, it is easily seen from (3.11) and (5.4) that $\left\|\eta_{n}\right\|=O\left(\tau^{3}\right)+O\left(\tau h^{2}\right)$, and so the convergence result follows from Lemma 5.1 and Remark 5.3.

In conclusion, it can be said that the parallel alternating scheme (1.7) does what it is expected to do: its temporal order of convergence is 2 , which is one higher than for the basic scheme. Moreover, the scheme (1.7) is exact in time for stationary solutions (so, in Tables 5.1-5.3 all errors would be 0 for (1.7)).

\section{SOME ALTERNATIVE FORMS}

The choice of the time levels used in (1.4) seems somewhat arbitrary. We can consider the more general formula, with parameter $c$,

$$
\begin{gathered}
u_{n+1 / 2}=u_{n}+\tau F_{1}\left(t_{n}+c \tau, \frac{1}{2} u_{n}+\frac{1}{2} u_{n+1 / 2}\right), \\
u_{n+1}=u_{n+1 / 2}+\tau F_{2}\left(t_{n}+(1-c) \tau, \frac{1}{2} u_{n+1 / 2}+\frac{1}{2} u_{n+1}\right) .
\end{gathered}
$$

When using (6.1) as a basic scheme (1.5), the alternating schemes (1.6), (1.7) are of 2 nd order in the classical ODE sense for any choice of $c$. Apart from $c=\frac{1}{4}$, which was used in (1.4), the choices $c=0$ or $c=\frac{1}{2}$ are also natural ones. Below it will be shown, however, that taking $c \neq \frac{1}{4}$ will cause a global order reduction for the parallel alternating scheme if the boundary values $\mathbf{u}_{\Gamma}$ are not constant in time.

In the same way as in $\S 3$, by using suitable perturbations $p_{k}, q_{k}$, it can be 
shown that (6.1) has local errors

$$
\begin{aligned}
\delta_{n}= & \left(I-\frac{1}{2} \tau A_{1}\right)^{-1}\left(I-\frac{1}{2} \tau A_{2}\right)^{-1} \\
& \times\left[\left(\frac{1}{2}-c\right) \tau^{2} \dot{v}_{h}\left(t_{n+1 / 2}\right)-\frac{1}{4} \tau^{2} A v_{h}\left(t_{n+1 / 2}\right)\right. \\
& \quad+\left(c-\frac{1}{4}\right) \tau^{2}\left(A_{1}-A_{2}\right) \dot{u}_{h}\left(t_{n+1 / 2}\right)+\left(c-\frac{1}{4}\right) \tau^{3} A_{1} A_{2} \dot{u}_{h}\left(t_{n+1 / 2}\right) \\
& \left.\quad+\left(\frac{1}{4} c-\frac{1}{8}\right) \tau^{3}\left(A_{1}-A_{2}\right) \dot{v}_{h}\left(t_{n+1 / 2}\right)+\left(\frac{1}{2} c^{2}-\frac{3}{4} c+\frac{1}{8}\right) \tau^{3} A \ddot{u}_{h}\left(t_{n+1 / 2}\right)\right] \\
& +O\left(\tau^{3}\right)+O\left(\tau h^{2}\right) .
\end{aligned}
$$

The local error of the parallel alternating scheme (1.7), with (6.1) as basic scheme, is now seen to be

$$
\begin{aligned}
& \sigma_{n}=\left(I-\frac{1}{2} \tau A_{1}\right)^{-1}\left(I-\frac{1}{2} \tau A_{2}\right)^{-1} \\
& \times\left[\left(c-\frac{1}{4}\right) \tau^{3} A_{1} A_{2} \dot{u}_{h}\left(t_{n+1 / 2}\right)+\left(\frac{1}{4} c-\frac{1}{8}\right) \tau^{3}\left(A_{1}-A_{2}\right) \dot{v}_{h}\left(t_{n+1 / 2}\right)\right. \\
& \left.+\left(\frac{1}{2} c^{2}-\frac{3}{4} c+\frac{1}{8}\right) \tau^{3} A \ddot{u}_{h}\left(t_{n+1 / 2}\right)\right] \\
& +O\left(\tau^{3}\right)+O\left(\tau h^{2}\right) .
\end{aligned}
$$

In case the boundary conditions for $\mathbf{u}$ are constant in time, it follows that $\dot{\mathbf{u}}=0$ on $\Gamma$ and $\tau^{3} A_{1} A_{2} \dot{u}_{h}(t)=O\left(\tau^{3}\right)$. The term involving $\tau^{3} A_{1} A_{2} \dot{u}_{h}\left(t_{n+1 / 2}\right)$ will lead, however, to a lower order of temporal convergence for time-dependent boundary conditions.

Example 6.1. Consider (2.1) with $\mathbf{g} \equiv 1$ and solution

$$
\mathbf{u}(x, y, t)=t \text {. }
$$

Then $\dot{u}_{h}(t)=e \otimes e$ and $v_{h}(t)=(2 \theta-1) e \otimes e$. Hence,

$$
\sigma_{n}=\left(c-\frac{1}{4}\right) \tau^{3}\left(I-\frac{1}{2} \tau A_{1}\right)^{-1}\left(I-\frac{1}{2} \tau A_{2}\right)^{-1} A_{1} A_{2}[e \otimes e] .
$$

This can be written as

$$
\sigma_{n}=\left(c-\frac{1}{4}\right) \tau^{3}\left[\left(I+\frac{1}{2} \tau Q\right)^{-1} Q e \otimes\left(I+\frac{1}{2} \tau Q\right)^{-1} Q e\right] .
$$

Consequently,

$$
\left\|\sigma_{n}\right\|=\left|c-\frac{1}{4}\right| \tau^{3}\left|\left(I+\frac{1}{2} \tau Q\right)^{-1} Q e\right|^{2} \geq\left|c-\frac{1}{4}\right| C^{2} \tau^{3 / 2}
$$

for some $C>0$, provided that $\tau / h^{2} \neq o(1)$ (see Lemma 2.2).

Since $\sigma_{n}$ is the error introduced in one step, it is clear that the order of convergence is $\leq \frac{3}{2}$ if $c \neq \frac{1}{4}$. In fact, it can be shown that there will be convergence with order 1 exactly when $\tau=h$. We shall not prove this result, since it is of little relevance here. The important thing is simply that timedependent boundary conditions should be treated in (6.1) with $c=\frac{1}{4}$. This choice was also used in [14].

One can also derive LOD methods starting from the trapezoidal rule

$$
u_{n+1}=u_{n}+\frac{1}{2} \tau F\left(t_{n}, u_{n}\right)+\frac{1}{2} \tau F\left(t_{n+1}, u_{n+1}\right)
$$

instead of the implicit midpoint rule (1.3). An LOD method of this type is given by

$$
\begin{aligned}
& u_{n+1 / 2}=u_{n}+\frac{1}{2} \tau F_{1}\left(t_{n}, u_{n}\right)+\frac{1}{2} \tau F_{1}\left(t_{n}+\frac{1}{2} \tau, u_{n+1 / 2}\right) \\
& u_{n+1}=u_{n+1 / 2}+\frac{1}{2} \tau F_{2}\left(t_{n}+\frac{1}{2} \tau, u_{n+1 / 2}\right)+\frac{1}{2} \tau F_{2}\left(t_{n+1}, u_{n+1}\right) .
\end{aligned}
$$


For strongly nonlinear problems, scheme (1.4) might possess better stability properties (cf. [14]), but for linear problems with constant coefficients, (6.3) will also be stable.

An error recursion $\varepsilon_{n+1}=R \varepsilon_{n}+\delta_{n}, \varepsilon_{n}=u_{h}\left(t_{n}\right)-u_{n}$, can be obtained by considering along with (6.3) a perturbed version

$$
\begin{aligned}
& \tilde{u}_{n+1 / 2}=\tilde{u}_{n}+\frac{1}{2} \tau F_{1}\left(t_{n}, \tilde{u}_{n}\right)+\frac{1}{2} \tau F_{1}\left(t_{n}+\frac{1}{2} \tau, \tilde{u}_{n+1 / 2}\right)+q_{n}, \\
& \tilde{u}_{n+1}=\tilde{u}_{n+1 / 2}+\frac{1}{2} \tau F_{2}\left(t_{n}+\frac{1}{2} \tau, \tilde{u}_{n+1 / 2}\right)+\frac{1}{2} \tau F_{2}\left(t_{n+1}, \tilde{u}_{n+1}\right)+q_{n+1 / 2} .
\end{aligned}
$$

By taking $\tilde{u}_{k}=u_{h}\left(t_{k}\right)$ for $k=n, n+\frac{1}{2}$ and $n \geq 0$, we get

$$
\begin{aligned}
& q_{n}=-\frac{1}{2} \tau v_{h}\left(t_{n+1 / 2}\right)+\frac{1}{8} \tau^{2} \dot{v}_{h}\left(t_{n+1 / 2}\right)+O\left(\tau^{3}\right)+O\left(\tau h^{2}\right), \\
& q_{n+1 / 2}=\frac{1}{2} \tau v_{h}\left(t_{n+1 / 2}\right)+\frac{1}{8} \tau^{2} \dot{v}_{h}\left(t_{n+1 / 2}\right)+O\left(\tau^{3}\right)+O\left(\tau h^{2}\right) .
\end{aligned}
$$

In the same way as in $\S 3$ (see the formulas (3.4), (3.5)), it follows that the local discretization error for scheme (6.3) applied to (2.1) is given by

$$
\begin{aligned}
\delta_{n}= & \left(I-\frac{1}{2} \tau A_{1}\right)^{-1}\left(I-\frac{1}{2} \tau A_{2}\right)^{-1} \\
& \times\left[\frac{1}{4} \tau^{2} \dot{v}_{h}\left(t_{n+1 / 2}\right)-\frac{1}{4} \tau^{2} A v_{h}\left(t_{n+1 / 2}\right)+\frac{1}{16} \tau^{3}\left(A_{2}-A_{1}\right) \dot{v}_{h}\left(t_{n+1 / 2}\right)\right] \\
& +O\left(\tau^{3}\right)+O\left(\tau h^{2}\right) .
\end{aligned}
$$

For the parallel alternating scheme based on (6.3) we get a local error

$$
\begin{aligned}
\sigma_{n}= & \left(I-\frac{1}{2} \tau A_{1}\right)^{-1}\left(I-\frac{1}{2} \tau A_{2}\right)^{-1}\left[\frac{1}{16} \tau^{3}\left(A_{2}-A_{1}\right) \dot{v}_{h}\left(t_{n+1 / 2}\right)\right] \\
& +O\left(\tau^{3}\right)+O\left(\tau h^{2}\right) .
\end{aligned}
$$

It can be seen, by following the previous proofs, that all error bounds for (1.5)-(1.7) remain unchanged if (6.3) is used as the basic scheme. The error structure is even somewhat simpler with (6.3).

\section{CONCLUDING REMARKS}

Of the three schemes (1.5), (1.6), and (1.7) considered in this paper, it is clear that the sequentially alternating scheme (1.6) is unsuited in its present form (i.e., without boundary corrections). The basic scheme (1.5) is 1st-order accurate in time. For some practical problems, 1st-order accuracy is sufficient, but even in such a situation it seems better to use instead of (1.5) the LOD method based on backward Euler, which has stronger damping properties and is also of 1 st order. Rather general convergence results for this LOD method were presented in [11].

For problems where more accuracy is demanded, the parallel alternating scheme (1.7) of [14], based on either (1.4) or (6.1), seems a good candidate. An alternative would be, for example, the Peaceman-Rachford ADI method. An analysis for this method can be found in [6]. Some numerical results given in [14] suggest that this ADI method and (1.7) are competitive.

A popular technique for improving accuracy is Richardson extrapolation. However, when both $\tau$ and $h$ tend to 0 , the structure of the temporal errors is different than for the classical ODE case $\tau \downarrow 0, h=h_{0}>0$. Therefore, it is not clear yet whether extrapolation will increase the order of convergence in general. Some interesting results in this direction have been derived in [1] 
for the LOD method based on backward Euler. Numerical results can also be found in [16].

\section{ACKNOWLEDGMENT}

The author is grateful to J. Blom for providing the numbers in Tables 5.1-5.3.

\section{BIBLIOGRAPHY}

1. W. Auzinger, On error structures and extrapolation for stiff systems with applications in the method of lines, Computing 44 (1990), 331-356.

2. P. Brenner, M. Crouzeix, and V. Thomée, Single step methods for inhomogeneous linear differential equations in Banach space, RAIRO Numer. Anal. 16 (1982), 5-26.

3. M. Fiedler, Special matrices and their applications in numerical mathematics, Martinus Nijhoff, Dordrecht, 1986.

4. P. J. van der Houwen and J. G. Verwer, One-step splitting methods for semi-discrete parabolic equations, Computing 22 (1979), 291-309.

5. W. H. Hundsdorfer and B. I. Steininger, Convergence of linear multistep and one-leg methods for stiff nonlinear initial value problems, BIT 31 (1991), 124-143.

6. W. H. Hundsdorfer and J. G. Verwer, Stability and convergence of the Peaceman-Rachford ADI method for initial-boundary value problems, Math. Comp. 53 (1989), 81-101.

7. P. Lancaster and M. Tismenetsky, The theory of matrices, 2 nd ed., Academic Press, Orlando, FL, 1985.

8. R. J. LeVeque, Intermediate boundary conditions for $L O D, A D I$ and approximate-factorization methods, ICASE report 85-21, NASA Langley Research Centre, Hampton, VA, 1985.

9. G. I. Marchuk, Methods of numerical mathematics, 2nd ed., Springer-Verlag, New York, 1981.

10. __ Splitting and alternating direction methods, Handbook of Numerical Analysis, vol. 1 (P. G. Ciarlet and J. L. Lions, eds.), North-Holland, Amsterdam, 1990, pp. 197-462.

11. A. A. Samarskii, On an economical difference method for the solution of a multidimensional parabolic equation in an arbitrary region, Zh. Vychisl. Mat. i Mat. Fiz. 2 (1962), 894-926.

12. J. M. Sanz-Serna, J. G. Verwer, and W. H. Hundsdorfer, Convergence and order reduction of Runge-Kutta schemes applied to evolutionary partial differential equations, Numer. Math. 50 (1987), 405-418.

13. B. P. Sommeijer, P. J. van der Houwen, and J. G. Verwer, On the treatment of timedependent boundary conditions in splitting methods for parabolic differential equations, Internat. J. Numer. Math. Engrg. 17 (1981), 335-346.

14. D. A. Swayne, Time dependent boundary and interior forcing in locally one-dimensional schemes, SIAM J. Sci. Statist. Comput. 8 (1987), 755-767.

15. J. G. Verwer, Contractivity of locally one-dimensional splitting methods, Numer. Math. 44 (1984), 247-259.

16. J. G. Verwer and H. B. de Vries, Global extrapolation of a first order splitting method, SIAM J. Sci. Statist. Comput. 6 (1985), 771-780.

17. N. N. Yanenko, The method of fractional steps, Springer-Verlag, Berlin, 1971.

Centre for Mathematics and Computer Science, P.O. Box 4079, 1009 AB Amsterdam, THE NETHERLANDS

E-mail address: willem@cwi.nl 